\title{
Chinese patients with Lewy body dementia had shorter survival and developed complications earlier than those with Alzheimer's disease
}

Yat-Fung Shea ${ }^{1}$, FHKAM, FHKCP, Shui-Ching $\underline{L e}^{1}$, BHS, Alex Chun Keung Shum ${ }^{2}$, FHKAM, FHKCP, Patrick Ka-Chun $\underline{\text { Chiu }}^{1}$, FHKAM, FHKCP, Leung-Wing $\underline{\operatorname{Chu}}^{1,3}$, MD, FRCP, Hon Wai Felix $\underline{\text { Chan }}^{1}$, FRCP, FHKAM

\begin{abstract}
INTRODUCTION Alzheimer's disease (AD) and Lewy body dementia (LBD) are two common forms of dementia. There are still controversies regarding whether LBD patients have a worse clinical course than AD patients.

METHODS We retrospectively reviewed all biomarkers that supported AD and LBD patients presenting to the Memory Clinic of Queen Mary Hospital, Hong Kong, between 1 January 2008 and 30 December 2016. Diagnoses of AD and LBD were supported by clinical diagnostic criteria and biomarkers. LBD patients included those with dementia with Lewy bodies (DLB) and Parkinson's disease dementia (PDD). Baseline demographics, presenting clinical features, degree of cognitive impairment and specified clinical outcomes were compared.

RESULTS We recruited $31 \mathrm{AD}$ and 25 LBD patients (18 DLB, 7 PDD). When measured from disease onset, LBD patients were noted to have shorter overall survival $(p=0.02)$ with earlier occurrence of falls $(p<0.001)$, dysphagia $(p<0.001)$, pneumonia $(p=0.01)$, pressure injuries $(p=0.003)$ and institutionalisation $(p=0.03)$ than AD patients. Cox regression analyses showed that LBD predicted falls (hazard ratio [HR] 5.86, 95\% confidence interval [Cl] 2.29-15.01, $p<0.001$ ), dysphagia (HR 10.06, 95\% Cl 2.50-40.44, $p=0.001$ ), pressure injuries (HR 17.39, 95\% Cl 1.51-200.10, $p=0.02$ ), institutionalisation ( $\mathrm{HR} 2.72,95 \% \mathrm{Cl} 1.12-6.60, \mathrm{p}=0.03$ ) and death (HR 2.96, 95\% Cl 1.18-7.42, $\mathrm{p}=0.02)$.

CONCLUSION LBD patients had shorter overall survival with earlier occurrence of pre-specified long-term events compared with AD patients. LBD also independently predicted pre-specified long-term events.
\end{abstract}

Keywords: Alzheimer's disease, dementia with Lewy bodies, Lewy body dementia, memory clinic, Parkinson's disease dementia

\section{INTRODUCTION}

With the increasing ageing of the Hong Kong population, more patients will be suffering from dementia. Alzheimer's disease $(A D)$ is the most common form of dementia, followed by vascular dementia and Lewy body dementia (LBD). ${ }^{(1,2)}$ LBD includes dementia with Lewy bodies (DLB) and Parkinson's disease dementia (PDD), which are differentiated based on the one-year rule of the relationship between motor manifestations (i.e. parkinsonism) and dementia (i.e. cognitive impairment). ${ }^{(3)}$ To facilitate diagnosis of the different types of dementia, cerebrospinal fluid (CSF) biomarkers, ${ }^{(4)}$ magnetic resonance (MR) imaging, ${ }^{(5)}$ functional imaging $\left({ }^{18} \mathrm{~F}\right.$-2-fluoro-2-deoxy-D-glucose positron emission tomography $\left[{ }^{18} \mathrm{FDG}-\mathrm{PET}\right]$ or single-photon emission computed tomography [SPECT]) and amyloid imaging (e.g. Pittsburgh Compound B [PIB]) are clinically utilised. ${ }^{(2)}$

In addition to an accurate diagnosis of the subtypes of dementia, caregivers are also concerned about the natural course of the different types of dementia. ${ }^{(3)}$ Serious problems for dementia include increased risk of falls, dysphagia, aspiration pneumonia, pressure injuries, caregiver stress leading to institutionalisation and long-term risk of mortality. ${ }^{(3)}$ Given the lack of definitive treatments for the various types of dementia, it remains prudent for clinicians to educate caregivers on the natural course of the various types of dementia so that they can be better prepared to acquire the necessary skills to care for their relatives, minimise long-term complications and even consider advance care planning at an earlier stage of the disease. ${ }^{(6)}$ Our previous study of 23 LBD patients with diagnoses supported by functional imaging found that falls, dysphagia, aspiration pneumonia, pressure injuries and death occurred in $70 \%, 52 \%, 26 \%, 26 \%$ and $30 \%$ of dementia patients, respectively. ${ }^{(3)}$

There have been a few studies comparing the clinical course of AD and LBD using fluid or imaging biomarker support that focused on outcomes such as admission, mortality and institutionalisation. ${ }^{(7-9)}$ In general, DLB patients were reported to have shorter survival with earlier admission and institutionalisation compared with AD patients. ${ }^{(7-9)}$ However, no difference in survival has been reported when survival was measured from the time of reported disease onset compared to from the time of diagnosis. ${ }^{(10)}$ To the best of our knowledge, there has not been any study among Chinese subjects that explores the differences in clinical course of $A D$ and LBD. The primary objective of this study was to compare the proportions and time of development of long-term complications, including falls, dysphagia, pneumonia, pressure injuries, institutionalisation and death, between two different types of dementia. The secondary objective was to compare the baseline clinical features, including cognitive symptoms and behavioural and psychological symptoms of dementia (BPSD).

${ }^{1}$ Department of Medicine, LKS Faculty of Medicine, University of Hong Kong, Queen Mary Hospital, ${ }^{2}$ Geriatric Division, Department of Medicine and Geriatrics, Tuen Mun Hospital, ${ }^{3}$ Alzheimer's Disease Research Network, Strategic Research Theme on Aging, University of Hong Kong, Hong Kong

Correspondence: Dr Shea Yat-Fung, Associate Consultant, Division of Geriatrics, Department of Medicine, LKS Faculty of Medicine, University of Hong Kong, Queen Mary Hospital, Hong Kong 102 Pokfulam Road, Hong Kong, Hong Kong SAR. elphashea@gmail.com 
We hypothesised that, compared with AD patients, LBD patients had shorter survival and suffered from more falls and pneumonia, with a shorter time to falls, pneumonia and institutionalisation.

\section{METHODS}

This was a retrospective observational study. The research protocol was approved by the Institutional Review Board of the University of Hong Kong/Hospital Authority, Hong Kong (UW17-483), and the requirement for informed consent was waived. We performed a retrospective review of the clinical records of all patients who presented to the memory clinic of Queen Mary Hospital between 1 January 2008 and 30 December 2016. All subjects had at least one year of clinical follow-up. AD patients fulfilled the latest diagnostic criteria for AD from the National Institute on Aging and Alzheimer's Association Research Framework, ${ }^{(11)}$ with either CSF examination showing AD pattern of CSF biomarkers (i.e. low amyloid-beta, and elevated total tau and phosphorylated tau), or ${ }^{11} \mathrm{C}-\mathrm{PIB}$ and ${ }^{18} \mathrm{FDG}$-PET showing bilateral temporoparietal hypometabolism and positive amyloid loading on ${ }^{11} \mathrm{C}$-PIB (i.e. binding occurred in more than one cortical brain region, i.e. frontal, parietal, temporal or occipital).. ${ }^{(1)}$ Subjects with LBD, which included those with DLB and PDD, fulfilled the diagnostic criteria of possible or probable DLB according to the fourth consensus report of the DLB consortium, or PDD for which dementia occurs more than one year after the established Parkinson's disease; ${ }^{(12)}$ the ${ }^{18} \mathrm{FDG}$-PET or SPECT images of these patients showed either reduced occipital metabolic activities or perfusion. ${ }^{(2)}$

We retrospectively corrected the clinical information, including age of onset of cognitive symptoms, age of presentation to the memory clinic, age of death, symptom duration, education level, gender, presenting scores of the Cantonese version of the Mini-Mental State Examination (C-MMSE), ${ }^{(13)}$ Clinical Dementia Rating, ${ }^{(14)}$ age-adjusted Charlson comorbidity index, ${ }^{(15)}$ initial presenting cognitive symptoms and BPSD according to the Neuropsychiatric Inventory scores. ${ }^{(16)}$ Age of presentation was defined as the age at which the patient first presented to the memory clinic. Symptoms duration was defined as the time between the first appearance of clinical symptoms of neurodegenerative disease and the first presentation to the memory clinic. Age of onset was defined as the difference between the age of presentation and symptoms duration. Disease duration was defined as the difference between age of onset and age of death. We also recorded the subjects' medication usage after the diagnosis was established.

Long-term outcomes, including falls, dysphagia, pneumonia, development of pressure injuries and mortality, were traced. Time to event was defined as the difference between onset of clinical symptoms and first appearance of these long-term events. In addition, time to events from the time of first presentation was calculated. A fall was defined as an event that results in the patient or a body part of the patient coming to rest inadvertently on the ground or other surface lower than the body. The date and the number of falls were recorded.

A diagnosis of pneumonia was made according to the following criteria: clinical signs and symptoms; white cell count of at least $10 \times 10^{9} / \mathrm{L}$ or a proportion of neutrophils of at least $80 \%$; fever (body temperature of at least $37.6^{\circ} \mathrm{C}$ ); and new infiltrates or consolidations on chest radiography (X-ray or computed tomography). The date and total number of pneumonia events were recorded.

Dysphagia was further sub-classified into oral and pharyngeal dysphagia according to the clinical assessment of a speech therapist or, if available, the report of video fluoroscopic swallowing studies. Penetration was defined as barium material entering the airway but not passing below the vocal cords, while aspiration was defined as barium material passing below the level of the vocal cords. ${ }^{(17)}$ The date of diagnosis of dysphagia was recorded. The location of pressure injuries and staging according to the National Pressure Ulcer Advisory Panel was also collected, ${ }^{(18)}$ and the date of discovery of pressure injuries recorded.

Institutionalisation was defined as placement in a residential institution regardless of the level of care (e.g. personal care facility, health care facility). The date of institutionalisation was traced as far back as possible, and the date of death was recorded.

Continuous variables with normal distribution were expressed as mean \pm standard deviation, or median with interquartile range. Categorical variables were studied using chi-square or Fisher's exact test, while continuous variables were compared using MannWhitney $U$ test or independent $t$-test as appropriate. Kaplan-Meier analyses were performed to study long-term event-free survival. Log-rank test was used to compare survival between AD and LBD patients. Analyses were censored at death or the last follow-up. Multivariate Cox regression analyses (enter model) were performed with each long-term clinical event as dependent variables and LBD vs. AD grouping, age of onset/presentation, symptoms duration, presenting MMSE, and antipsychotic usage as independent variables. A two-tailed $p$-value $<0.05$ indicated statistical significance. All statistical analyses were carried out using IBM SPSS Statistics version 24.0 for Windows (IBM Corp, Armonk, NY, USA).

\section{RESULTS}

There were a total of 31 AD patients and 25 LBD patients (18 DLB and 7 PDD). A comparison of the presenting baseline demographics is summarised in Table I. There were no significant differences in age of onset, age of presentation, comorbidities (as reflected by age-adjusted Charlson comorbidity index) or the degree of cognitive impairment (as reflected by presenting C-MMSE and Clinical Dementia Rating). There were significantly greater proportions of female patients in the AD group than the LBD group (61.3\% vs. $28.0 \%, p=0.04)$ and patients with higher education in the LBD group than the AD group (mean 7.8 \pm 4.7 vs. $1.6 \pm 1.0, p<0.001$ ). There was no significant difference in follow-up duration or ever-usage of acetylcholinesterase inhibitors or anti-psychotics between the two groups of patients.

The key clinical features of LBD were more frequent in the LBD group than the AD group, including fluctuation of consciousness ( $36 \%$ vs. $0 \%, \mathrm{p}<0.001)$, Parkinsonism $(72 \%$ vs. $0 \%, p<0.001)$, recurrent visual hallucination $(76 \%$ vs. $6.5 \%$, $\mathrm{p}<0.001)$ and rapid eye movement sleep behavioural disorder 
Table I. Comparison of clinical characteristics between AD patients and LBD patients.

\begin{tabular}{|c|c|c|c|}
\hline \multirow[t]{2}{*}{ Characteristic } & \multicolumn{2}{|c|}{ Mean \pm SD } & \multirow[t]{2}{*}{ p-value } \\
\hline & $A D(n=31)$ & LBD $(n=25)$ & \\
\hline Age of onset (yr) & $74.3 \pm 10.5$ & $73.9 \pm 6.1$ & $0.84^{\mathrm{a}}$ \\
\hline Age of presentation $(\mathrm{yr})$ & $77.3 \pm 9.9$ & $76.4 \pm 6.3$ & $0.60^{\mathrm{a}}$ \\
\hline Disease duration* $(y r)$ & $5.9(4.0-9.3)(n=8)$ & $7.0(3.8-9.0)(n=14)$ & $0.70^{\mathrm{b}}$ \\
\hline Age of death & $84.9 \pm 7.7(n=8)$ & $80.2 \pm 7.5(n=14)$ & $0.18^{\mathrm{a}}$ \\
\hline Female $^{\dagger}$ & $19(61.3)$ & $7(28.0)$ & $0.04^{c}$ \\
\hline Years of education & $1.6 \pm 1.0$ & $7.8 \pm 4.7$ & $<0.001^{\mathrm{a}}$ \\
\hline Presenting C-MMSE & $17.0 \pm 7.3$ & $18.0 \pm 8.0$ & $0.83^{\mathrm{a}}$ \\
\hline Presenting CDR* & $1(0.5-2.0)$ & $1(0.5-1.5)$ & $0.62^{\mathrm{b}}$ \\
\hline Age-adjusted CCI & $5.0 \pm 2.0$ & $5.0 \pm 1.2$ & $0.44^{\mathrm{a}}$ \\
\hline Duration of follow-up (mth) & $63.0 \pm 29.0$ & $54.5 \pm 35.4$ & $0.33^{\mathrm{a}}$ \\
\hline Ever usage of AChEl during follow-up ${ }^{\dagger}$ & $6(19.4)$ & $4(16.0)$ & $1.00^{c}$ \\
\hline Ever usage of anti-psychotic during follow-up ${ }^{\dagger}$ & $16(51.6)$ & $17(68.0)$ & $0.22^{c}$ \\
\hline
\end{tabular}

Data presented as *median (interquartile range) and tno. (\%). alndependent t-test; ${ }^{\mathrm{b}}$ Mann-Whitney $U$ test; ${ }^{\mathrm{c}} \mathrm{chi}$-square test. AChEl: acetylcholinesterase inhibitor; AD: Alzheimer's disease; C-MMSE: Mini-Mental State Examination (Cantonese version); CCI:Charlson comorbidity index; CDR: Clinical Dementia Rating; LBD: Lewy body dementia

Table II. Comparison of long-term clinical outcomes between AD and LBD patients.

\begin{tabular}{|c|c|c|c|}
\hline \multirow[t]{2}{*}{ Studied outcomes } & \multicolumn{2}{|c|}{ No. (\%)/mean \pm SD } & \multirow[t]{2}{*}{ p-value } \\
\hline & $A D(n=31)$ & LBD $(n=25)$ & \\
\hline \multicolumn{4}{|l|}{ Falls } \\
\hline Suffered from falls & $7(22.6)$ & $18(72.0)$ & $<0.001^{*}$ \\
\hline No. of falls & $0.4 \pm 0.8$ & $1.2 \pm 1.1$ & $0.002^{+}$ \\
\hline \multicolumn{4}{|l|}{ Dysphagia } \\
\hline $\begin{array}{l}\text { Suffered from } \\
\text { dysphagia }\end{array}$ & 4 (12.9) & $13(52.0)$ & $0.003^{*}$ \\
\hline $\begin{array}{l}\text { Suffered from oral } \\
\text { dysphagia }\end{array}$ & $4(12.9)$ & $12(48.0)$ & $0.007^{*}$ \\
\hline $\begin{array}{l}\text { Suffered from } \\
\text { pharyngeal dysphagia }\end{array}$ & $4(12.9)$ & $12(48.0)$ & $0.007^{*}$ \\
\hline \multicolumn{4}{|l|}{ Pneumonia } \\
\hline $\begin{array}{l}\text { Suffered from } \\
\text { pneumonia }\end{array}$ & $5(16.1)$ & $10(40.0)$ & $0.045^{*}$ \\
\hline $\begin{array}{l}\text { No. of pneumonia } \\
\text { events }\end{array}$ & $0.4 \pm 1.0$ & $0.8 \pm 1.4$ & $0.18^{+}$ \\
\hline Pressure injuries & $2(6.5)$ & $7(28.0)$ & $0.06^{*}$ \\
\hline Institutionalisation & $10(32.3)$ & $13(52.0)$ & $0.14^{*}$ \\
\hline Death & $8(25.8)$ & $14(56.0)$ & $0.02^{*}$ \\
\hline $\begin{array}{l}\text { Any one of the above } \\
\text { outcomes }\end{array}$ & $16(51.6)$ & $23(92.0)$ & $0.001^{*}$ \\
\hline
\end{tabular}

*Chi-square test. †Independent sample t-test. AD: Alzheimer's disease; LBD: Lewy body dementia; SD: standard deviation

( $52 \%$ vs. $0 \%$, p < 0.001). Otherwise, the other cognitive symptoms did not show significant differences between the two groups of patients. For a comparison of neuropsychiatric scores, LBD patients scored higher in hallucination subscores (median 1 vs. 0 , $\mathrm{p}=0.01$ ) and anxiety subscores (median 1 vs. $0, \mathrm{p}=0.01$ ) than AD patients. AD patients scored higher in agitation subscores than LBD patients, although the median scores did not numerically differ between the two groups $(p=0.03)$.

A comparison of long-term clinical outcomes between AD and LBD patients is summarised in Table II. Proportionately more LBD patients suffered from falls $(72.0 \%$ vs. $22.6 \%, \mathrm{p}<0.001)$, with a greater number of falls (mean $1.2 \pm 1.1$ vs. $0.4 \pm 0.8, p=0.002$ ), dysphagia $(52.0 \%$ vs. $12.9 \%, p=0.003)$ - including both oral and pharyngeal dysphagia, pneumonia $(40.0 \%$ vs. $16.1 \%, p=0.045)$ and mortality $(56.0 \%$ vs. $25.8 \%, p=0.02)$. The results of the Kaplan-Meier analysis are summarised in Fig. 1. LBD patients were noted to have earlier occurrence of falls $(p<0.001)$, dysphagia $(p<0.001)$, pneumonia $(p=0.01)$, pressure injuries $(p=0.003)$ and institutionalisation $(p=0.03)$ than AD patients when measured from disease onset; however, the results were similar if they were measured from presentation (Fig. 2). Similarly, LBD patients had shorter survival than AD patients if the survival rate was measured from disease onset $(p=0.02)$, but there was a trend of shorter survival for LBD patients if the survival rate was measured from presentation ( $p=0.06$; Figs. $1 \& 2$ ).

When measured from disease onset, Cox regression analyses showed that the LBD group independently predicted a higher risk of falls (hazard ratio [HR] 5.86, 95\% confidence interval [Cl] 2.29-15.01, p < 0.001), dysphagia (HR 10.06, 95\% Cl 2.50-40.44, $\mathrm{p}=0.001)$, pressure injuries (HR 17.39, 95\% Cl 1.51-200.10, $\mathrm{p}=0.02)$, institutionalisation $(\mathrm{HR} 2.72,95 \% \mathrm{Cl}$ 1.12-6.60, $\mathrm{p}=0.03)$ and death (HR 2.96, 95\% Cl 1.18-7.42, $\mathrm{p}=0.02$ ) than the AD group (Table III). In addition, there was interaction between LBD and dysphagia, which further increased the risk of pneumonia ( $\mathrm{HR} 8.32,95 \% \mathrm{Cl} 2.42-28.62, \mathrm{p}=0.001$ ). When analysed from presentation, the LBD group had a higher risk of falls (HR 45.60, 95\% Cl 2.04-1020.10, p=0.02) and dysphagia (HR 27.68, 95\% Cl 1.22-627.09, $\mathrm{p}=0.04$ ), after adjustment of covariates (Table III).

\section{DISCUSSION}

It is difficult to make a clinical differentiation between $\mathrm{AD}$ and LBD, especially in the absence of parkinsonism, and there is also a lot of overlap in their clinical symptoms, ${ }^{(19)}$ as noted in our study. Such difficulty is most likely due to the common co-existence of $\mathrm{AD}$ and $\mathrm{LBD}$ pathologies; for instance, $50 \%$ of DLB patients are amyloid-positive on amyloid PET scan. ${ }^{(20)}$ Clinically, it is 

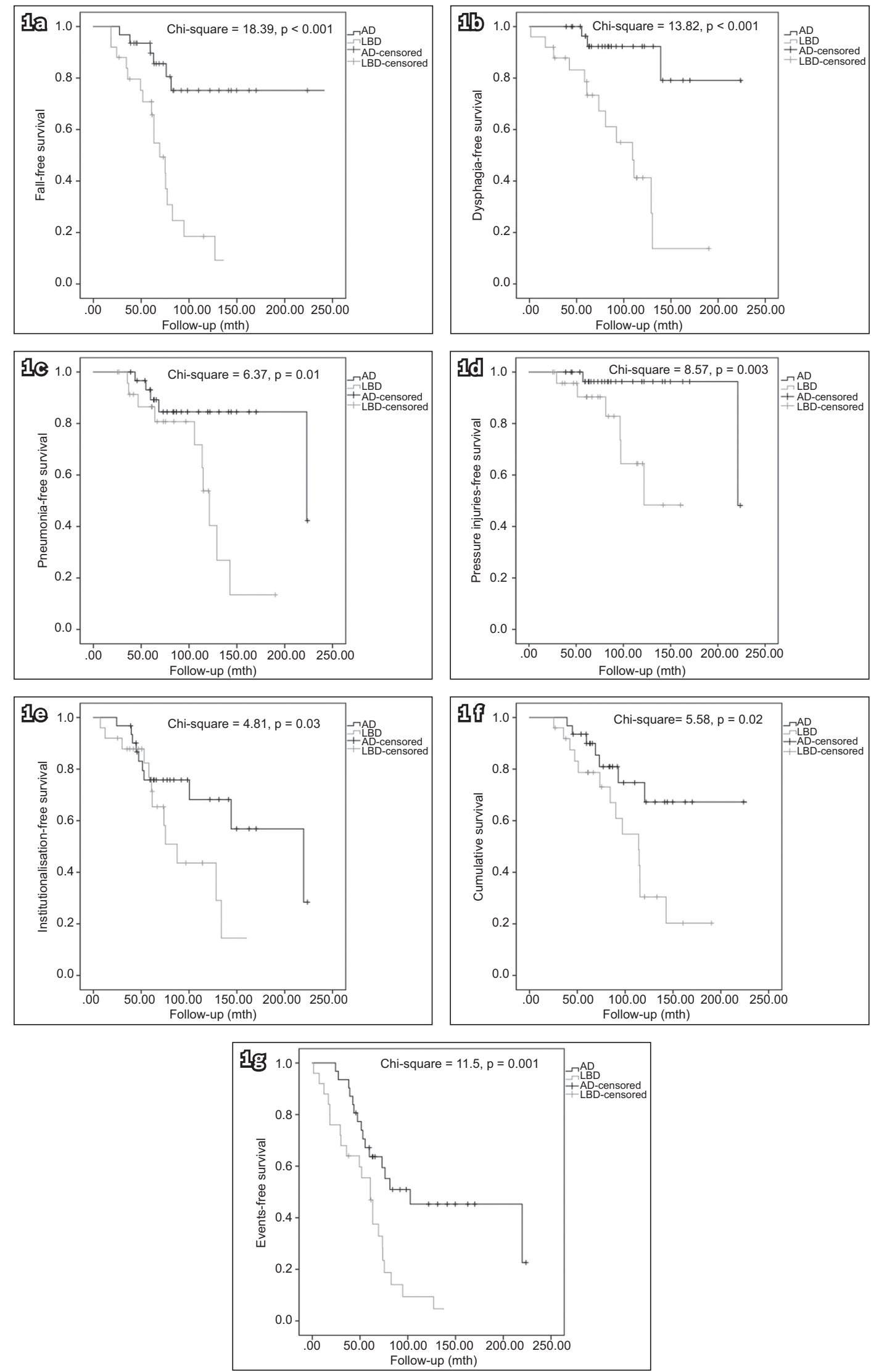

Fig. 1 Kaplan-Meier survival curves (measured from onset) show (a) fall-free survival; (b) dysphagia-free survival; (c) pneumonia-free survival; (d) pressure injuriesfree survival; (e) institutionalisation-free survival; (f) cumulative survival; and (g) long-term event-free survival. AD: Alzheimer's disease; LBD: Lewy body dementia. 

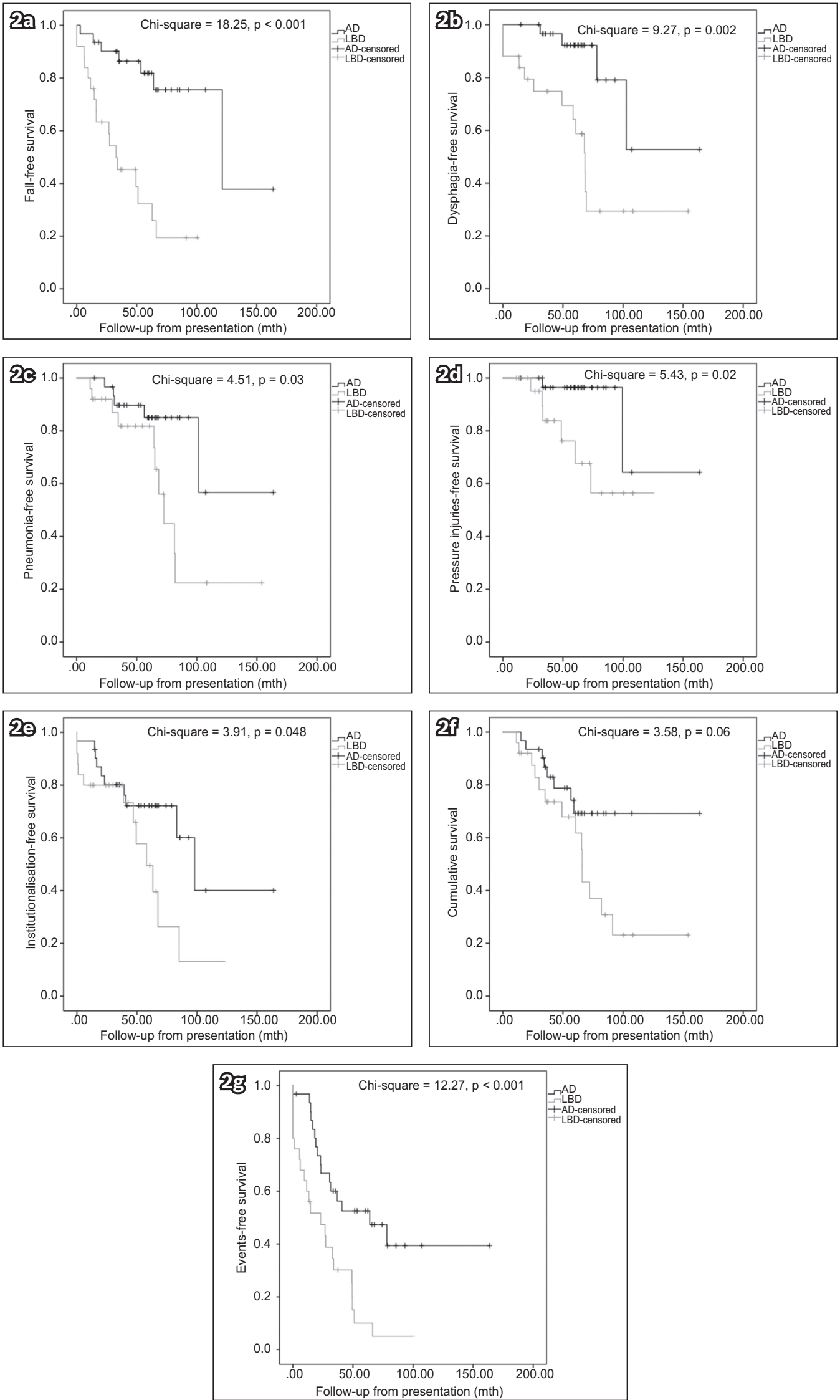

Fig. 2 Kaplan-Meier survival curves (measured from presentation) show (a) fall-free survival; (b) dysphagia-free survival; (c) pneumonia-free survival; (d) pressure injuries-free survival; (e) institutionalisation-free survival; (f) cumulative survival; and (g) long-term event-free survival. AD: Alzheimer's disease; LBD: Lewy body dementia. 
Table III. Cox regression analyses of various long-term events from onset and presentation.

\begin{tabular}{|c|c|c|c|c|}
\hline \multirow[t]{2}{*}{ Outcome/covariable } & \multicolumn{2}{|c|}{ From onset } & \multicolumn{2}{|c|}{ From presentation } \\
\hline & HR $(95 \% \mathrm{Cl})$ & p-value & HR (95\% CI) & p-value \\
\hline \multicolumn{5}{|l|}{ Falls } \\
\hline LBD vs. AD & $5.86(2.29-15.01)$ & $<0.001$ & $45.60(2.04-1,020.10)$ & 0.02 \\
\hline Antipsychotic usage & $0.70(0.20-2.43)$ & 0.58 & $1.95(0.24-15.62)$ & 0.53 \\
\hline Age of onset/presentation & $1.03(0.97-1.10)$ & 0.30 & $0.84(0.72-0.96)$ & 0.01 \\
\hline C-MMSE & $1.01(0.95-1.07)$ & 0.87 & $0.94(0.84-1.05)$ & 0.26 \\
\hline Symptoms duration & - & - & $0.72(0.53-0.99)$ & 0.045 \\
\hline \multicolumn{5}{|l|}{ Dysphagia } \\
\hline LBD vs. AD & $10.06(2.50-40.44)$ & 0.001 & $27.68(1.22-627.09)$ & 0.04 \\
\hline Antipsychotic usage & $2.25(0.67-7.53)$ & 0.19 & $4.61(0.59-35.79)$ & 0.14 \\
\hline Age of onset/presentation & $0.97(0.90-1.05)$ & 0.44 & $0.86(0.75-0.99)$ & 0.03 \\
\hline C-MMSE & $0.99(0.93-1.06)$ & 0.77 & $0.91(0.78-1.05)$ & 0.18 \\
\hline Symptoms duration & - & - & $0.46(0.26-0.81)$ & 0.007 \\
\hline \multicolumn{5}{|l|}{ Pneumonia } \\
\hline LBD vs. AD & $1.80(0.35-9.12)$ & 0.48 & $0.26(0.01-5.79)$ & 0.40 \\
\hline Dysphagia & $7.12(1.60-31.75)$ & 0.01 & $1.77(0.21-14.75)$ & 0.60 \\
\hline LBD + dysphagia* & $8.32(2.42-28.62)$ & 0.001 & $0.82(0.16-4.30)$ & 0.81 \\
\hline Antipsychotic usage & $0.81(0.15-4.35)$ & 0.81 & $0.28(0.01-5.67)$ & 0.28 \\
\hline Age of onset/presentation & $0.98(0.91-1.06)$ & 0.63 & $0.89(0.79-1.02)$ & 0.09 \\
\hline C-MMSE & $1.04(0.95-1.13)$ & 1.04 & $0.97(0.86-1.10)$ & 0.60 \\
\hline Symptoms duration & - & - & $0.72(0.49-1.05)$ & 0.72 \\
\hline \multicolumn{5}{|l|}{ Pressure injuries } \\
\hline LBD vs. AD & $17.39(1.51-200.10)$ & 0.02 & $5.75(0.21-155.49)$ & 0.30 \\
\hline Antipsychotic usage & $19.41(2.41-156.10)$ & 0.005 & $11.54(0.39-339.88)$ & 0.16 \\
\hline Age of onset/presentation & $0.97(0.84-1.11)$ & 0.66 & $0.93(0.79-1.09)$ & 0.35 \\
\hline C-MMSE & $1.18(0.96-1.45)$ & 0.12 & $1.08(0.86-1.35)$ & 0.54 \\
\hline Symptoms duration & - & - & $0.82(0.53-1.28)$ & 0.39 \\
\hline \multicolumn{5}{|l|}{ Institutionalisation } \\
\hline LBD vs. AD & $2.72(1.12-6.60)$ & 0.03 & $11.54(0.38-349.56)$ & 0.16 \\
\hline Antipsychotic usage & $1.81(0.72-4.56)$ & 0.21 & $15.86(0.63-400.85)$ & 0.09 \\
\hline Age of onset/presentation & $1.01(0.96-1.07)$ & 0.72 & $0.87(0.73-1.03)$ & 0.10 \\
\hline C-MMSE & $1.10(0.92-1.31)$ & 0.68 & $0.91(0.75-1.09)$ & 0.29 \\
\hline Symptoms duration & - & - & $0.99(0.74-1.33)$ & 0.96 \\
\hline \multicolumn{5}{|l|}{ Death } \\
\hline LBD vs. AD & $2.96(1.18-7.42)$ & 0.02 & $0.69(0.20-2.33)$ & 0.55 \\
\hline Usage of antipsychotics & $1.61(0.57-4.53)$ & 0.37 & $1.16(0.39-3.47)$ & 0.79 \\
\hline Age of onset/presentation & $1.04(0.98-1.11)$ & 0.20 & $1.03(0.95-1.11)$ & 0.50 \\
\hline C-MMSE & $0.97(0.91-1.03)$ & 0.97 & $0.94(0.87-1.03)$ & 0.20 \\
\hline Symptoms duration & - & - & $0.74(0.58-0.96)$ & 0.02 \\
\hline
\end{tabular}

*Interaction between LBD and dysphagia. AD: Alzheimer's disease; Cl: confidence interval; C-MMSE: Mini-Mental State Examination (Cantonese version); LBD: Lewy body dementia; HR: hazard ratio

important to differentiate between the two conditions because of differences in clinical management (e.g. typical antipsychotics are contraindicated in LBD patients due to the risk of neuroleptic hypersensitivity) and clinical symptoms (e.g. LBD patients have more vivid visual hallucination and autonomic symptoms such as postural hypotension). (21) From our study, fluctuation of consciousness, visual hallucination, rapid eye movement sleep behavioural disorder and parkinsonism (i.e. according to current clinical diagnostic criteria) remain the ideal clinical markers for the differentiation of LBD and AD. Clinicians should also be aware that LBD patients suffer more frequently from anxiety during presentation. Stubendorff et al reported a higher usage of anxiolytics among DLB patients than AD patients. ${ }^{(8)}$

In the current study, more LBD patients suffered from falls, dysphagia and pneumonia than AD patients. Furthermore, LBD patients had earlier occurrence of falls, dysphagia, pneumonia, pressure injuries and institutionalisation than $\mathrm{AD}$ patients, when these were measured from disease onset, although the results 
Table IV. Comparison of characteristics and clinical outcomes of AD and LBD patients among studies. .7-10 $^{\text {a }}$

\begin{tabular}{|c|c|c|c|c|c|c|}
\hline \multirow[t]{2}{*}{ Characteristic/outcome } & \multicolumn{2}{|c|}{ Current study; 2018} & \multicolumn{2}{|c|}{ Stubendorff et al; $2011^{(8)}$} & & \\
\hline & $A D(n=31)$ & $\operatorname{LBD}^{a}(n=25)$ & $\begin{array}{l}\text { AD } \\
(n=79)\end{array}$ & $\begin{array}{l}\text { DLB } \\
(n=49)\end{array}$ & & \\
\hline Age of presentation* (yr) & $77.3 \pm 9.9$ & $76.4 \pm 6.3$ & $76.0 \pm 6.0$ & $75.8 \pm 6.0$ & & \\
\hline $\mathrm{MMSE}^{\dagger}$ & $17 \pm 7.3$ & $18 \pm 8.0$ & 21 & 22 & & \\
\hline Follow-up time ${ }^{\ddagger}$ (mth) & 63 & 54.5 & 78 & 56.4 & & \\
\hline \multicolumn{7}{|l|}{ Outcomes (\%) } \\
\hline Admission & - & - & - & - & & \\
\hline Fall & $22.6^{\S}$ & $72^{\S}$ & - & - & & \\
\hline Pneumonia & $16.1^{\S}$ & $40^{5}$ & - & - & & \\
\hline Institutionalisation & $32.3^{\S}$ & $52^{\S}$ & - & - & & \\
\hline $\begin{array}{l}\text { Time to nursing home after } \\
\operatorname{diagnosis}^{\dagger}(y r)\end{array}$ & $2.9 \pm 2.6$ & $4.1 \pm 3.2$ & - & - & & \\
\hline Death & $25.8^{\S}$ & $56^{\S}$ & 63 & 77 & & \\
\hline \multicolumn{7}{|l|}{ Time to death ${ }^{c, \neq}(y r)$} \\
\hline From presentation & 3.1 & 4.1 & $6.5^{\S}$ & $4.7^{\S}$ & & \\
\hline From onset & $7.6^{\S}$ & $6.8^{\S}$ & 9.4 & 7.7 & & \\
\hline Clinical diagnosis & \multicolumn{2}{|c|}{$\begin{array}{l}\text { Further supported by a combination of CSF } \\
\text { biomarkers, }{ }^{18} \mathrm{FDG}-\mathrm{PET},{ }^{11} \mathrm{C}-\mathrm{PIB} \text { or SPECT }\end{array}$} & \multicolumn{2}{|c|}{$\begin{array}{l}\text { Further supported by CSF } \\
\text { biomarkers }\end{array}$} & & \\
\hline \multirow[t]{2}{*}{ Characteristic/outcome } & \multicolumn{2}{|c|}{ Hanyu et al; 2009(9) } & \multicolumn{2}{|c|}{ Williams et al; 2006 ${ }^{(7)}$} & \multicolumn{2}{|c|}{ Walker et al; $2000^{(10)}$} \\
\hline & $A D(n=111)$ & DLB $(n=56)$ & $\begin{array}{l}A D \\
(n=252)\end{array}$ & $\begin{array}{l}\text { DLB } \\
(n=63)\end{array}$ & $\begin{array}{l}\text { AD } \\
(n=39)\end{array}$ & $\begin{array}{l}\text { DLB } \\
(n=31)\end{array}$ \\
\hline Age of presentation* (yr) & $77.5 \pm 6.2$ & $78.1 \pm 5.2$ & $77.8 \pm 9.5^{\S}$ & $73.5 \pm 8.7^{\S}$ & $78.1^{\mathrm{b}}$ & $75.8^{\mathrm{b}}$ \\
\hline MMSE $^{\dagger}$ & $20.3 \pm 3.7$ & $20.7 \pm 3.8$ & - & - & $15.8 \pm 7.6$ & $16.7 \pm 7.1$ \\
\hline Follow-up time ${ }^{\ddagger}$ (mth) & 52 & 40 & 75.6 & 60 & 36 & 36 \\
\hline \multicolumn{7}{|l|}{ Outcomes (\%) } \\
\hline Admission & $14^{\S}$ & $30^{5}$ & - & - & - & - \\
\hline Fall & 3 & 7 & - & - & - & - \\
\hline Pneumonia & & & - & - & - & - \\
\hline Institutionalisation & & & - & - & - & - \\
\hline $\begin{array}{l}\text { Time to nursing home after } \\
\text { diagnosis }^{\dagger}(y r)\end{array}$ & & & 6.6 & 6.1 & - & - \\
\hline Death & & & - & - & 48.8 & 53.1 \\
\hline \multicolumn{7}{|l|}{ Time to death ${ }^{c, \neq}(y r)$} \\
\hline From presentation & & & 6.3 & 5 & 3.1 & 3.5 \\
\hline From onset & & & $9.5^{\S}$ & $7.7^{\S}$ & 5.6 & 5.5 \\
\hline Clinical diagnosis & \multicolumn{2}{|c|}{$\begin{array}{l}\text { Further supported by SPECT and MIBG scan } \\
\text { (for DLB patients) }\end{array}$} & \multicolumn{2}{|c|}{$\begin{array}{l}\text { Based on post-mortem } \\
\text { examination }\end{array}$} & \multicolumn{2}{|c|}{ Based on clinical criteria } \\
\hline
\end{tabular}

Data presented as *mean \pm SD; tmedian or mean \pm SD; $\neq$ median or mean. $\$ p<0.05$. ${ }^{a}$ Consisted of 18 DLB and 7 Parkinson's disease dementia patients. ${ }^{b}$ Age of onset of dementia. 'Statistical analysis for survival difference by Kaplan-Meier curve and log-rank test. AD: Alzheimer's disease; ${ }^{11} \mathrm{C}-\mathrm{PIB}:{ }^{11} \mathrm{C}-\mathrm{Pittsburgh}$ Compound B; CSF cerebrospinal fluid; DLB: Dementia with Lewy bodies; ${ }^{18} \mathrm{FDG}-\mathrm{PET}$ : ${ }^{18} \mathrm{~F}-2$-fluoro-2-deoxy-D-glucose positron emission tomography; LBD: Lewy body dementia; MIBG: metaiodobenzylguanidine; MMSE: Mini-Mental State Examination; SPECT: single-photon emission computed tomography

remained similar when measured from presentation. When measured from disease onset, LBD independently predicted a higher risk of falls, dysphagia, pressure injuries, institutionalisation and death (only falls and dysphagia when measured from presentation). The higher risks of falls, pressure injuries and institutionalisation are likely related to parkinsonism, and impaired balance and mobility in LBD patients. ${ }^{(21)}$ The higher risks of dysphagia and pneumonia are likely attributed to impairment of the swallowing mechanism in LBD. ${ }^{(22)}$ The more frequent appearance of dysphagia and pneumonia likely contributes to higher mortality among these patients. Hanyu et al reported a higher proportion of death among DLB patients compared to AD patients (30\% vs. $14 \%$, p < 0.05). ${ }^{(9)}$ Therefore, primary caregivers of LBD patients should be instructed on how to minimise the risk of falls (e.g. more attention to environmental risk factors) and the proper ways of feeding in cases of dysphagia (e.g. use of thickener or postural adjustment like chin-tug), ${ }^{(22)}$ and be made aware of the early features of pneumonia as well as the need for frequent turning in case immobilisation becomes a problem. These long-term clinical issues should also be discussed during advanced care planning sessions. ${ }^{(6)}$

Western studies have reported shorter survival for DLB patients, although such findings have not been replicated in 
all studies. ${ }^{(7,8)}$ A comparison of the findings between our study and those of other studies with a similar design is summarised in Table IV. Stubendorff et al ${ }^{(8)}$ compared survival between AD $(n=79)$ and DLB $(n=49)$ patients from the reported disease onset and time of diagnosis, or based on MMSE scores, with clinical diagnosis of dementia further supported by CSF biomarkers for all AD patients and some DLB patients. They found that the survival time was significantly shorter in the DLB group compared with the $\mathrm{AD}$ group, both from the time of diagnosis and at cognitive levels of 20 and 17 on the MMSE; however, no difference in survival was found when measured from the reported disease onset. ${ }^{(8)}$ Williams et al, ${ }^{(7)}$ who studied the survival and mortality differences between DLB $(n=63)$ and AD $(n=252)$ patients with post-mortem confirmation of diagnosis, reported that DLB patients had an increased risk of mortality compared with $A D$ patients (HR 1.88, 95\% Cl 1.4-2.5). They also found that DLB patients had a shorter survival after dementia onset (7.3 vs. 8.5 years, $p<0.02){ }^{\text {(7) }}$ Walker et al, whose study involved 39 AD and 31 DLB patients who were diagnosed based on clinical criteria, noted no difference in the time to death measured from onset or presentation. ${ }^{(10)}$ The differences in findings among the above studies could be related to the possibilities of co-existing AD pathologies in LBD patients. ${ }^{(20)}$ Our study provides more evidence of shorter survival for LBD patients when measured from disease onset.

There are several limitations to our study. First, the sample size was small compared to some of the previous similar studies, which was also reflected by the wide $\mathrm{Cl}$ in the $\mathrm{HR}$ of Cox regression analyses and the infrequent occurrence of certain complications. Second, there was no post-mortem pathological examination performed and, therefore, the possibility for mixed pathologies or other aetiologies of neurodegenerative disease could not be ruled out. Third, our study was performed in Chinese patients who presented to a single memory clinic, thus limiting the generalisability of the results. Fourth, the study design was retrospective in nature, which may have resulted in bias being introduced during data collection, especially with regard to the presence or absence of symptoms or long-term clinical events, which depended on documentation in clinical records. Further, there could also be recall bias, since the time of symptoms onset was based on primary caregivers' recall. Finally, the severity of parkinsonism was not measured quantitatively, and there was also missing data, which renders it impossible to compare the rate of cognitive decline. Moreover, the apolipoprotein E statuses of our subjects were not available although it is a well-known risk factor for cognitive decline, influencing time to events. Despite these limitations, our study is the first to compare AD and DLB among Chinese patients and will provide useful clinical information for local clinical practice.
In summary, the current study showed that LBD patients presented more commonly with hallucination and anxiety. When measured from disease onset, LBD patients had shorter overall survival and earlier occurrence of long-term events, including falls, dysphagia, pneumonia, pressure injuries and institutionalisation, as compared with AD patients. LBD also independently predicted these long-term complications when measured from disease onset.

\section{REFERENCES}

1. Alzheimer's Association. 2016 Alzheimer's disease facts and figures. Alzheimers Dement 2016; 12:459-509.

2. Shea YF, Ha J, Lee SC, Chu LW. Impact of (18)FDG PET and (11)C-PIB PET brain imaging on the diagnosis of Alzheimer's disease and other dementias in a regional memory clinic in Hong Kong. Hong Kong Med J 2016; 22:327-33.

3. Shea YF, Chu LW, Lee SC. A descriptive study of Lewy body dementia with functional imaging support in a Chinese population: a preliminary study. Hong Kong Med J 2017; 23:222-30.

4. Shea YF, Chu LW, Zhou L, et al. Cerebrospinal fluid biomarkers of Alzheimer's disease in Chinese patients: a pilot study. Am J Alzheimers Dis Other Demen 2013; 28:769-75.

5. Shea YF, Ha J, Chu LW. Progressive supranuclear palsy presenting initially as semantic dementia. J Am Geriatr Soc 2014; 62:2459-60.

6. Luk JKH, Chan FHW. End-of-life care for advanced dementia patients in residential care home-a Hong Kong perspective. Ann Palliat Med 201 8; 7:359-64.

7. Williams MM, Xiong C, Morris JC, Galvin JE. Survival and mortality differences between dementia with Lewy bodies vs Alzheimer disease. Neurology 2006; 67:1935-41.

8. Stubendorff K, Hansson O, Minthon L, Londos E. Differences in survival between patients with dementia with Lewy bodies and patients with Alzheimer's disease-measured from a fixed cognitive level. Dement Geriatr Cogn Disord 2011; 32:408-16.

9. Hanyu H, Sato T, Hirao K, et al. Differences in clinical course between dementia with Lewy bodies and Alzheimer's disease. Eur J Neurol 2009; 16:212-7.

10. Walker Z, Allen RL, Shergill S, Mullan E, Katona CL. Three years survival in patients with a clinical diagnosis of dementia with Lewy bodies. Int J Geriatr Psychiatry 2000; 15:267-73.

11. Jack CR Jr, Bennett DA, Blennow K, et al. NIA-AA Research Framework: toward a biological definition of Alzheimer's disease. Alzheimers Dement 2018; 14:535-62.

12. McKeith IG, Boeve BF, Dickson DW, et al. Diagnosis and management of dementia with Lewy bodies: fourth consensus report of the DLB Consortium. Neurology 2017; 89:88-100

13. Chiu HFK, Lee HC, Chung WS, Kwong PK. Reliability and validity of the Cantonese version of the Mini-Mental State Examination: a preliminary study. J Hong Kong Coll Psychiatr 1994; 4:25-8.

14. Morris JC. The Clinical Dementia Rating (CDR): current version and scoring rules. Neurology 1993; 43:2412-4.

15. Charlson ME, Pompei $P$, Ales KL, MacKenzie CR. A new method of classifying prognostic comorbidity in longitudinal studies: development and validation. J Chronic Dis 1987; 40:373-83.

16. Cummings JL, Mega M, Gray K, et al. The Neuropsychiatric Inventory: comprehensive assessment of psychopathology in dementia. Neurology 1994; 44:2308-14

17. Rosenbek JC, Robbins JA, Roecker EB, Coyle JL, Wood JL. A penetrationaspiration scale. Dysphagia 1996; 11:93-8.

18. National Pressure Ulcer Advisory Panel. NPUAP Pressure Injury Stages. Available at: https://npuap.org/page/2014Guidelines. Accessed August 28, 2019.

19. Rizzo G, Arcuti S, Copetti M, et al. Accuracy of clinical diagnosis of dementia with Lewy bodies: a systematic review and meta-analysis. J Neurol Neurosurg Psychiatry 2018; 89:358-66.

20. Ossenkoppele R, Jansen WJ, Rabinovici GD, et al. Prevalence of amyloid PET positivity in dementia syndromes: a meta-analysis. JAMA 2015; 313:1939-49.

21. Walker Z, Possin KL, Boeve BF, Aarsland D. Lewy body dementias. Lancet 2015; 386:1683-97.

22. Luk JK, Chan DK. Preventing aspiration pneumonia in older people: do we have the 'know-how'? Hong Kong Med J 2014; 20:421-7. 